\title{
Time-course of cortical networks involved in working memory
}

\author{
Phan Luu 1,2 *, Daniel M. Caggiano ${ }^{3+}$, Alexandra Geyer ${ }^{3}$, Jenn Lewis ${ }^{1,2}$, Joseph Cohn $^{4}$ and \\ Don M. Tucker ${ }^{1,2}$
}

1 Electrical Geodesics, Inc., Eugene, OR, USA

${ }^{2}$ Department of Psychology, University of Oregon, Eugene, OR, USA

${ }^{3}$ Aptima, Inc., Woburn, MA, USA

${ }^{4}$ Office of Naval Research, Arlington, VA, USA

\section{Edited by:}

Shuhei Yamaguchi, Shimane

University, Japan

\section{Reviewed by:}

Theodore Zanto, University of California San Francisco, USA Joseph Allen Harris,

Otto-von-Guericke Universität

Magdeburg, Germany

\section{*Correspondence:}

Phan Luu, Electrical Geodesics, Inc. 1600 Millrace Drive, Eugene, OR 97403, USA

e-mail: pluu@egi.com

${ }^{\dagger}$ Present address:

Daniel M. Caggiano, Battelle

Memorial Institute, Arlington, VA, USA
Working memory (WM) is one of the most studied cognitive constructs. Although many neuroimaging studies have identified brain networks involved in WM, the time course of these networks remains unclear. In this paper we use dense-array electroencephalography (dEEG) to capture neural signals during performance of a standard WM task, the $n$-back task, and a blend of principal components analysis and independent components analysis (PCA/ICA) to statistically identify networks of WM and their time courses. Results reveal a visual cortex centric network, that also includes the posterior cingulate cortex, that is active prior to stimulus onset and that appears to reflect anticipatory, attention-related processes. After stimulus onset, the ventromedial prefrontal cortex, lateral prefrontal prefrontal cortex, and temporal poles become associated with the prestimulus network. This second network appears to reflect executive control processes. Following activation of the second network, the cortices of the temporo-parietal junction with the temporal lobe structures seen in the first and second networks re-engage. This third network appears to reflect activity of the ventral attention network involved in control of attentional reorientation. The results point to important temporal features of network dynamics that integrate multiple subsystems of the ventral attention network with the default mode network in the performance of working memory tasks.

\section{Keywords: dense-array EEG, working memory, attention, temporal parietal junction, frontal lobe}

\section{INTRODUCTION}

An essential capacity for cognition and human performance is working memory (WM), the ability to hold information in store as it is manipulated through various cognitive transformations. The $n$-back task, which has been in use at least since 1958 (Kirchner, 1958), can easily accommodate working load manipulations and different stimulus features. This property allowed it to be used to study WM across various sensory modalities, and a number of early fMRI studies used $n$-back tasks to isolate and compare the neural mechanisms for maintaining and manipulating items in WM. Although not specifically developed to isolate component processes of WM (see Jaeggi et al., 2010), the body of evidence that has developed through the use of the $n$-back task is impressive, not only in the sheer number of published studies but also in what it has revealed about brain regions and networks responsible for WM. In a meta-analytic study, Owen et al. (2005) identified brain regions that are commonly engaged in WM, as assessed by the $n$-back task, and they include: premotor, cingulate, lateral prefrontal, fronto-polar, and medial and lateral posterior parietal cortices. In addition, activations often are observed in perceptual representation-specific regions, including inferior temporal and lateral occipital cortices (Druzgal and D'Esposito, 2001). However, nothing is known about the time course(s) of these brain regions and networks in WM.
In the event-related potential (ERP) literature, Gevins et al. (1996) conducted the first WM ERP studies with the n-back task. Using dense-array electroencephalography (dEEG), these authors observed early- (200 ms post-stimulus), mid- (390 ms post-stimulus), and late-latency (600-900 ms post-stimulus) components that differed as a function of WM load. Specifically, early activity was larger for high load conditions, whereas activity at the mid- and late-latency intervals was attenuated for high WM loads.

Using other visual WM paradigms designed to investigate specific WM processes, researchers have shown that activity $300 \mathrm{~ms}$ post-stimulus over occipitoparietal recordings sites is sensitive to memory maintenance requirements (e.g., Kiss et al., 1998; McCollough et al., 2007), memory updates (Kiss et al., 1998, 2007), and selection of memory traces for maintenance (Yi and Friedman, 2011). In contrast, frontal components at approximately $400 \mathrm{~ms}$ post-stimulus reflect either inhibition of irrelevant stimuli (Yi and Friedman, 2011) or the initiation of updates to the memory store (Kiss et al., 2007).

Studies that employ ERP methodology can leverage both appropriate experimental design and the inherent temporal properties of ERP features to infer functional significance. However, because the vast majority of ERP studies of WM have employed either sparse EEG arrays or analytic techniques such as surface Laplacian or highly simplistic head models that do not 
take advantage of modern advances in electro-magnetic imaging, information about the relevant underlying brain structures has been lacking. Furthermore, temporal overlap in activity among the underlying neural generators of scalp-recorded EEG can produce complicated scalp voltage distribution patterns that cloud the interpretation of traditionally measured, discrete ERP components (Dien et al., 2004). Consequently, findings from the ERP WM literature have not been integrated with fMRI findings.

Recently, neuroimaging studies have examined the dynamics among the cerebral nodes that support WM by assessing the functional connectivity within and among brain networks during $n$-back performance, leaving an even larger gap between the ERP and fMRI bodies of literature. fMRI functional connectivity studies have observed temporal correlations among the regions noted above during $n$-back performance that are consistent with "taskpositive" network (TPN) activity seen in a wide range of tasks (e.g., Fox et al., 2005). Although the specific nodes of this network vary slightly from study to study depending on the task, the method of assessing functional connectivity, and the seed regions used, TPN nodes responsive to $n$-back load typically include lateral prefrontal cortex (IPFC), the insula, the anterior cingulate cortex (ACC), and/or the supramarginal gyrus (SMG - Newton et al., 2011; Gordon et al., 2012; Sala-Llonch et al., 2012). The TPN is believed to support controlled attention processes (Fox et al., 2005), and stronger functional connectivity within the TPN during WM delays is associated with more accurate performance in WM tasks (Pessoa et al., 2002).

Several fMRI studies also have observed functional connectivity in the default mode network (DMN) reflecting task-induced, correlated deactivation among medial frontal and posterior cingulate cortices during $n$-back performance (Hampson et al., 2006; Newton et al., 2011; Gordon et al., 2012; Sala-Llonch et al., 2012). Activity within the DMN is also directly related to task performance. Individual differences in the DMN connectivity strength, whether assessed during a resting state or during task performance, predict individual differences in response accuracy (Hampson et al., 2006; Sala-Llonch et al., 2012).

Thus, while both TPN and DMN functional connectivity are correlated with behavioral performance, the way in which these two networks interact with one another and with other cortical nodes involved in specific aspects of cognition to produce effective task performance remains unclear. Above and beyond the degree to which TPN and DMN connectivity predict task performance individually, the strength of the anti-correlation between these two networks also predicts WM task performance (Hampson etal. (2006)). Furthermore, Chadick and Gazzaley (2011) demonstrated that activity within visual category-specific regions of inferior temporal cortex representing one of two attended stimulus categories (i.e., increased FFA activity when faces were attended and houses were ignored) correlated with activity in the task-positive network. Simultaneously, suppressed activity with regions representing the ignored stimulus category (i.e., decreased PPA activity when houses were ignored) correlated with activity in the default-mode network. While this study clearly demonstrates that the TPN and DMN must coordinate to support task performance in a way that goes beyond simple alternating activity among the two networks, little is known about how these two networks dynamically interact to support performance during a continuous WM task such as the $n$-back. However, understanding the way in which these networks interact to support task performance requires a far better understanding of the temporal dynamics of this interaction than is possible with fMRI.

In the present study, we employed dEEG methodology and an advanced head model for accurate source estimation to examine both the time course and cortical networks involved in WM, as assessed by the $n$-back task. We applied temporal principal components analysis (PCA) and spatial independent components analysis (ICA) to decompose the scalp-recorded brain activity. This analytic approach decomposes signals that overlap in time and space, with each principal component representing cortical activity that is temporally and spatially correlated. Each resulting component is independent from the others. These statistical decomposition techniques also improve source analysis because noise is separated and removed from the signal (Dien, 2012). The results allow us to delineate not only the underlying dynamics of WM networks but also their temporal courses, laying a foundation for future studies that employ more refined paradigms for further isolation of different WM processes.

\section{MATERIALS AND METHODS PARTICIPANTS}

Twenty-four right-handed participants from the general student population at the University of Oregon completed the study. Due to EEG data quality (two participants) and behavioral performance (five participants), data from seven participants were not analyzed further. Of the remaining 17 participants, 7 were males, and their age ranged between 19 and 60 (median $=22, \mathrm{SD}=12.4$ ). It is noted that three participants had age ranging from 57 to 60 . When these participants were removed (median $=22, \mathrm{SD}=3.3$ ), the significant behavioral and dEEG results reported below do not change. Therefore, we retained these three subjects in all analyses.

All participants had normal or corrected-to-normal vision and reported no history of seizures or loss of consciousness and no current medications or use of illicit drugs that could affect the EEG. The experimental protocol was approved by institutional review boards at EGI, the University of Oregon, and the Office of Naval Research. All subjects provided informed written consent prior and received $\$ 30$ for participating.

\section{STIMULI AND EXPERIMENTAL DESIGN}

Each stimulus (see Figure 1) consisted of two diagonal, overlapping wrenches (Weber et al., 1997; Caggiano and Parasuraman, 2004). In each stimulus, the two wrenches differed in shape (one contained a hexagonally shaped head and the other a C-shaped head) and in color (green or purple). Each stimulus was divided virtually into four quadrants; the head for each wrench appeared in one of these four quadrants in any given stimulus. The total set of stimuli included all possible combinations of wrench color, wrench head quadrant, and depth (which wrench was overlaid on top of the other), resulting in a set of 32 stimuli. Each stimulus from the total available set was used equally often across all experimental blocks. Participants viewed each stimulus at a distance of $65 \mathrm{~cm}$ from the monitor, and each stimulus subtended $9.5^{\circ} \times 9.5^{\circ}$. 

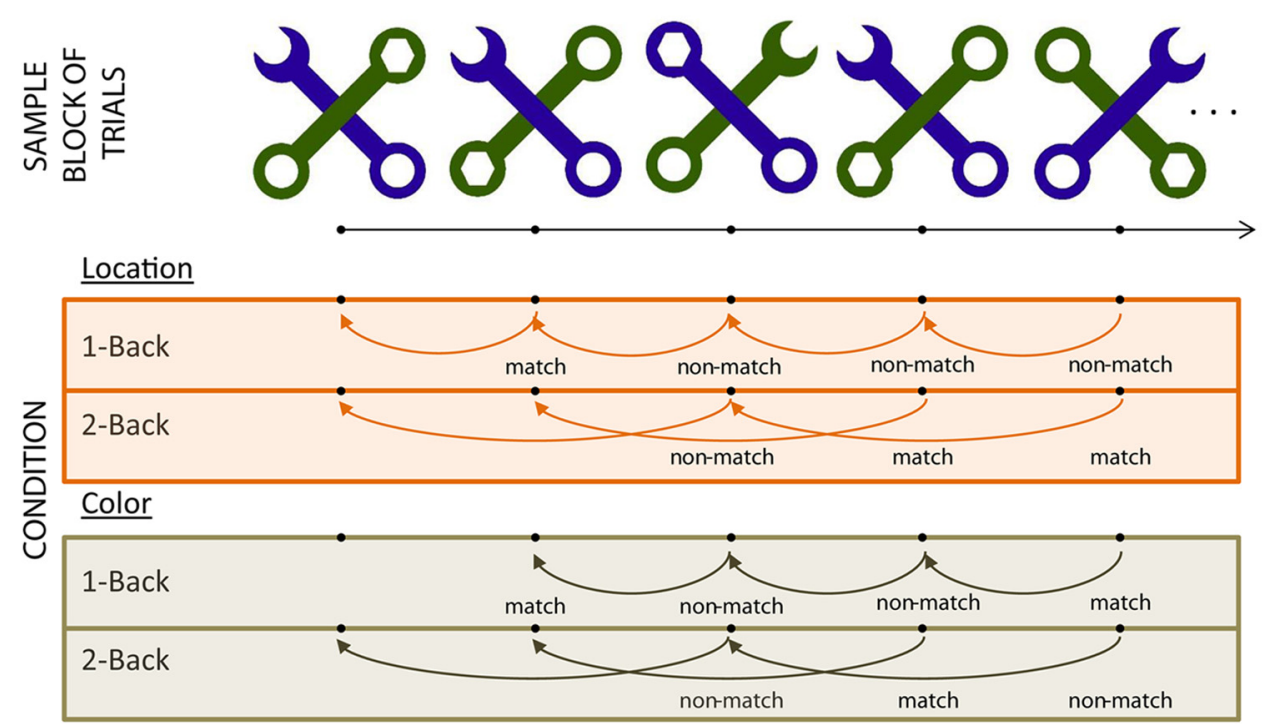

FIGURE 1 | Task schematic (see Stimuli and Experimental Design)

The experiment employed a 2 (WM load) by 2 (Task - color vs. location) blocked design. Participants indicated whether a key feature of the C-shaped wrench of each stimulus matched the similar feature on the stimulus presented " $n$ " trials previously by pressing a button on "match" trials (see Figure 1). In the "color" blocks, participants based their comparisons on the color of the C-shaped wrench. In "location" blocks, they based their comparisons on the stimulus quadrant in which the C-shaped wrench head appeared.

On each trial, the stimulus was presented for $2000 \mathrm{~ms}$ followed by a randomly jittered inter-stimulus interval of 1000 $1500 \mathrm{~ms}$. The experiment included two blocks of each of the WM load $\times$ Task conditions, resulting in a total of eight blocks. The order of blocks was randomized with respect to condition with the following constraints - the two blocks for any given condition always were run in succession (i.e., the first 1-back color block was always followed by the second 1-back color block). The study consisted of eight blocks; each block contained 128 trials and ran for approximately $8 \mathrm{~min}$. The condition order was counterbalanced across participants.

\section{EEG RECORDING}

Electroencephalography was acquired using a 256-channel HydroCel Geodesic Sensor Net (EGI, Eugene, OR, USA). All electrode impedances were kept below $70 \mathrm{~K} \Omega$ (Ferree et al., 2000). Recordings were referenced to Cz. The EEG was low-passed filtered $(100 \mathrm{~Hz})$ prior to being sampled at 250 samples/s with a 24-bit analog-to-digital converter.

\section{PROCEDURE}

After providing informed consent, participants were fitted with the 256-channel Hydrocel Geodesic Sensor Net (EGI, Eugene, OR, USA). To minimize head movements, participants rested their head on a chin rest during task performance. Once participants were fitted with the EEG Sensor Net, they received instructions for the first task that they would perform and were given 12 practice trials. In between blocks, participants took a short break, as needed. Participants viewed new instructions and performed 12 new practice trials every time they encountered a block with a new condition. The entire study lasted $3 \mathrm{~h}$.

\section{EEG PROCESSING}

The continuous EEG was band-pass filtered at $0.1-30 \mathrm{~Hz}$ with zero-phase shift FIR filters. The data were then segmented relative to the onset of each stimulus (200 ms before and $1000 \mathrm{~ms}$ after). Only correctly identified match trials were included in the analysis. Trials were then sorted according to task and load. Trials with blinks or ocular movement artifacts were excluded. Trials with more than 10 bad channels (defined as any sample that exceed a voltage threshold of $200 \mu \mathrm{V}$ or a sample-to-sample transition threshold of $100 \mu \mathrm{V}$ ) were also excluded. The data were then averaged and re-referenced to the average reference. The data were not baseline corrected prior to submission to the PCA/ICA procedures because we want to examine any WM related effects that precedes stimulus onset, as would be expected for the $n$-back task.

\section{PRINCIPAL COMPONENTS ANALYSIS}

Because the ERPs reflect superimposed activity from multiple sources with overlapping time courses, we decomposed the ERPs using PCA. The average data for each subject were entered into a temporo-spatial PCA using the ERP PCA Toolkit version 2.14 (Dien, 2010, 2012). In this analysis, a temporal decomposition is first performed on the covariance matrix with time points as the variables. The PCA factor structure was rotated using the Promax procedure, with subjects, conditions, and channels as the sources of variance. The temporal components thus reflect patterns of covariance among time points. Following the temporal PCA, spatial decomposition of each time course component was 
conducted with ICA, wherein channels were now the variables, and the factor structure was rotated to achieve spatial independent components with infomax. Because many components will be generated, our strategy for analysis of the components begins with elimination of all components that do not account for at least $0.5 \%$ of the variance. Next, we search for components that are identifiable as ERP components and have been previously reported to be affected by WM load manipulations. These components are tested for statistical reliability using repeated measures ANOVA (see Results section) without correction for multiple comparisons. For the remaining components, they were visually examined to determine if load effects are present. These analyses were performed with Bonferonni correction for multiple comparisons.

\section{SOURCE ESTIMATES}

Each temporospatial component represents multiple sources that functionally covary in time and space. Each PCA/ICA component reflects a static topography, and the only thing that varies is the amplitude of the components over time; analysis of the sources for each component at each time point produces the same source map, with source amplitude changing to reflect changes in the component amplitude. We hypothesize that each component describes activity from either a single brain structure or a network of brain structures that covary together over the task conditions and across individuals. Source estimates were conducted for load-sensitive components derived from the temporospatial PCA/ICA, which are statistically identified.

Sources were estimated using GeoSource (version 2.0) electrical source imaging software (EGI, Eugene, OR, USA). GeoSource uses a finite difference method (FDM) of head tissue conductivity for accurate computation of the lead field in relation to head tissues, where the primary resistive component is the skull. The FDM allows accurate characterization of the cranial orifices, primarily the optical canals and foramen magnum. Tissue compartments of the FDM were constructed from whole head MRI and CT scans of a single subject (Colin27) whose head shape closely matches the Montreal Neurological Institute (MNI) average MRI (MNI305). The MRI and CT images were co-registered prior to segmentation of the brain and cerebral spinal fluid (identified from MRI data), and the skull and scalp (identified from CT images), and the individual's MRI and CT images were aligned with the cortex volume from the MNI305 atlas with Talaraich registration. The tissue volumes were parceled into $2-\mathrm{mm}$ voxels to form the computational elements of the FDM. Conductivity values used in the FDM model are as follows: $0.25 \mathrm{~S} / \mathrm{m}$ for brain, $1.8 \mathrm{~S} / \mathrm{m}$ for cerebral spinal fluid, $0.018 \mathrm{~S} / \mathrm{m}$ for skull, and $0.44 \mathrm{~S} / \mathrm{m}$ for scalp (see Ferree et al., 2000). These values reflect recent evidence that the skull-to-brain conductivity ratio is about 1:14 (Ryynanen et al., 2006; Zhang et al., 2006), compared to the 1:80 ratio traditionally assumed.

Source locations were derived from the probabilistic map of the MNI305 average. Based on the probabilistic map, gray matter volume was parceled into 7-mm voxels; each voxel served as a source location with three orthogonal orientation vectors. This resulted in a total of 2,394 source triplets whose anatomic identities were derived through use of a Talaraich demon (Lancaster et al., 2000). Once the head model was constructed, an average of the 256-channel sensor positions was registered to the scalp surface. To compute estimates of the sources, a minimum norm solution with the LORETA constraint (Pascual-Marqui, 2002) was used. All source estimates were performed on the temporospatial components from the grand-averaged data.

\section{RESULTS}

\section{BEHAVIORAL DATA}

Of the 24 participants that completed the study, two participants were excluded from analysis due to excessive ocular artifacts. Data from five additional participants were excluded due to poor performance in at least one task condition. Of these five, three seemed to have confused the response mapping in one of the conditions; the other two were unable to reach a criterion level of performance (75\% accuracy).

The response time (RT) and accuracy data from the remaining 17 participants were entered into separate 2 (task: color vs. location) $\times 2$ (load: 1-back vs. 2 -back) repeated measures ANOVAs. The RT data indicated significant main effects of task $[F(1,16)=9.56, p=0.007, \mathrm{MSE}=10,554]-$ participants responded more quickly in the location (mean $=668 \mathrm{~ms}$ ) than in the color (mean $=745 \mathrm{~ms}$ ) conditions - and WM load $[F(1,16)=25.4, p<0.001, \mathrm{MSE}=11,997]$ - participants were faster in the 1 -back (mean $=640 \mathrm{~ms}$ ) than in the 2-back (mean $=774 \mathrm{~ms}$ ) conditions. The interaction between task and load was not significant in the RT data $[F(1,16)=0.564, p=0.464$, MSE $=3609]$. The accuracy data also indicated main effects of task $[F(1,16)=4.78, p=0.044, \mathrm{MSE}=0.002]$ and load $[F(1,16)=25.4, p<0.001, \mathrm{MSE}=0.003$, see Figure 2]. Participants responded significantly more accurately in the location $\left[\right.$ mean $_{\text {location }}=95.9 \%$, mean $_{\text {color }}=93.6 \%$ ] and in the 1 -back conditions $\left(\operatorname{mean}_{1-\text { back }}=98.2 \%\right.$, mean $\left._{2-\text { back }}=91.3 \%\right)$. In addition, a significant task $\times$ load interaction $[F(1,16)=5.84$, $p=0.028$, MSE $=0.001]$ indicated that the effect of load was larger for the color task (an $8.7 \%$ difference in accuracy) than for the location task (a 5\% difference in accuracy, see Figure 2).

\section{EVENT-RELATED POTENTIALS}

On visual inspection, several traditional ERP components appeared to be sensitive to WM load (Figure 3). At mediofrontal sites (at $\sim 350 \mathrm{~ms}$ ), a positive component was larger for the 2-back than the 1-back location condition. At approximately $400 \mathrm{~ms}$ over centroparietal sites, the late positive complex (LPC; i.e., P3) also differentiated between WM load, with 1-back location showing the largest amplitude (see Introduction, especially for the location task). Following the peak of the LPC, slow negative-going features of the ERP over frontal and posterior sites also differed as a function of load.

\section{Principal components analysis}

Temporal PCA decomposed the traditional ERP data into distinct temporal factors. A parallel test (Horn, 1965) was performed to determine the number of temporal factors to retain for the spatial decomposition step. In this test, a Scree plot is generated for a fully random dataset (with the same dimensionality as the actual 
A

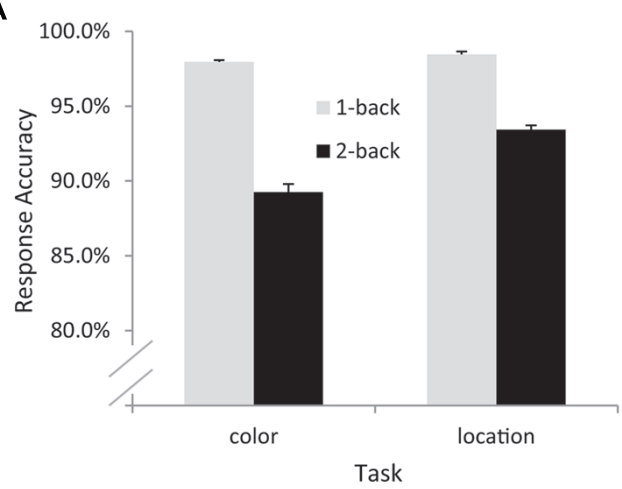

B

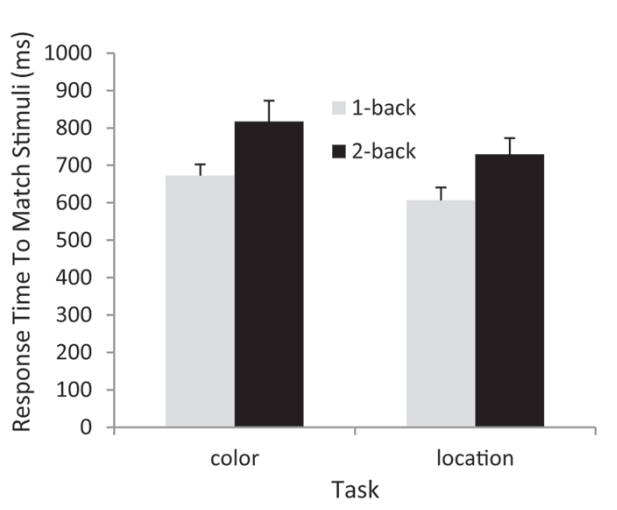

FIGURE 2 | Behavioral data from all participants included in the EEG analyses. Accuracy (A) and response time (B) data. Note that response time data are from correctly detected match trials only.
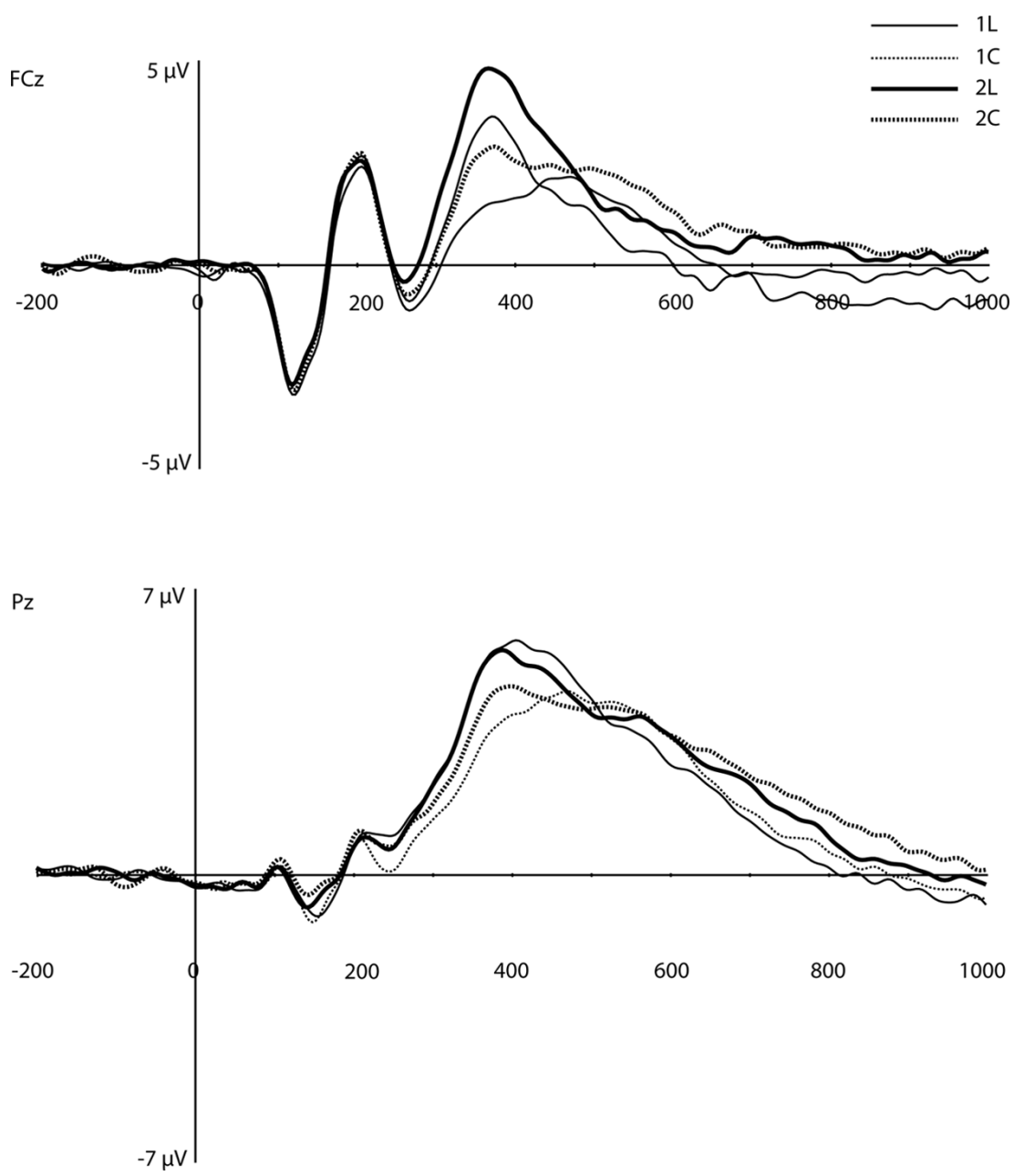

FIGURE 3 | Original grand-average ERP waveforms at FCz and Pz. Note that in this figure, a baseline correction (between -200 ms and stimulus onset) was applied, in contrast to all PCA/ICA components displayed in Figures 4-6. 
data set) for comparison with the Scree plot from the actual data. The point at which the two Scree plots cross indicates the number of factors to be retained. Based on the parallel test, eight factors, accounting for $93 \%$ of the variance, were retained. A second parallel test on the spatial ICA of the eight temporal factors indicated that four spatial factors, accounting for $78 \%$ of the total variance, should be retained for each temporal factor.

Of the 32 temporospatial factors recovered, we eliminated 21 components because they each accounted for less than $0.5 \%$ of the variance. Thus, we are left with 11 components for analysis. Applying our criterion of component resemblance to known ERP components (with regards to latency and scalp distribution) that have been shown in previous research to be affected by WM load, we were able to identify two components [Component 1 (C1) and Component 9 (C9), see below]. After this step 9 components remained for exploratory analysis, wherein Bonferroni correction (at 0.05) was applied.

Repeated measures ANOVAs were performed on the scalp voltage data at the peak channels and latency for each component, with Task and Load as within-subjects factors. Of the 11 components, three components showed statistically reliable differences in WM load. We present the analysis of each of these temporospatial components in the following sections.

\section{Component 1 (C1)}

The waveforms and scalp topography of $\mathrm{Cl}$ are illustrated in Figure 4. In this figure, the grand-average ERP waveforms (Figure 4A) are presented for all four conditions at the channel overlaying the location indicated in the topographic map (white dot in Figure 4B). C1 is characterized by load effects in both the pre-stimulus interval and in a post-stimulus interval that peaks at $456 \mathrm{~ms}$. This component is similar to the LPC, which includes the P3 and related components (see Dien et al., 2004), in its topography and time course in the poststimulus interval. This same temporospatial component, however, also seems to capture the pre-stimulus stimulus preceding negativity (SPN). Statistical tests were performed on two windows, defined through visual inspection, of C1 (-200 to $80 \mathrm{~ms}$, and 340-630 ms, highlighted in green in Figure 4A). We derived an average of each window re-referenced to the 130 $300 \mathrm{~ms}$ post-stimulus interval (where differences between the four waveforms were minimal). The data were then submitted to repeated-measures ANOVA with Task, Load, and Time (Pre-stimulus vs. Post-stimulus) as within-subjects factors. Note that because this component reflects the LPC/SPN components that have been noted to be affected by load manipulations (see Discussion), significance level was not adjusted for multiple comparisons.

The ANOVA revealed significant effects for Load, $F(1,16)=8.4$, $p<0.02$, Task, $F(1,16)=5.3, p<0.04$, Time $\times$ Load, $F(1,16)=8.4, p<0.02$, and Time $\times$ Task $(1,16)=5.3$, $p<0.04$ (see Table 1). The Time $\times$ Load effect indicated that although the amplitude was larger in the 1-back than in the 2-back condition in both time windows, the polarity of the component reversed from the pre-stimulus to the poststimulus window. Paired $t$-tests performed at each time interval revealed that the differences were significant $-t(16)=-2.9$, $p<0.02$ (pre-stimulus), $t(16)=2.9, p<0.02$ (post-stimulus). Similarly, inspection of the mean for the Time $\times$ Task effect showed that the location task was associated with greater amplitude overall, but the polarity of the component differed over time. Paired $t$-tests also confirmed that during the pre-stimulus interval the location task was associated with greater negative amplitudes than the color task, $t(16)=-2.3, p<0.04$, whereas the location task elicited larger positive amplitudes than the color task during the post-stimulus interval, $t(16)=2.3$, $p<0.04$.

In order to understand the pre-stimulus time course of $\mathrm{C} 1$ over a somewhat longer interval, we re-segmented the data with a window of $1000 \mathrm{~ms}$ (vs. the initial $200 \mathrm{~ms}$ ) before and 500 post-stimulus onset. Because more subjects blinked in the longer pre-stimulus interval, the number of participants with enough artifact free trials for signal averaging was reduced from 17 to 14 .

The same temporospatial PCA/ICA procedure was applied to this subset of subjects, and the parallel test revealed that 10 temporal factors, accounting for $94 \%$ of the total variance, should be retained for the next (spatial ICA) step. The parallel test for the spatial ICA step revealed four spatial components should be retained for each temporal component, accounting for $81 \%$ of the total variance. Examination of these components revealed a component with a similar time course, topography, and condition differences as C1 (Figures 4D,E). Consistent with the interpretation of an SPN, C1 activity in this longer interval began to deviate from baseline approximately $400 \mathrm{~ms}$ before stimulus onset (Figure 4D).

In considering the pre-stimulus activity for the $\mathrm{C} 1$ component, if this activity is in fact an SPN, reflecting functional engagement of visual association in preparation for the visual perception, then trials with a smaller C1 or SPN would be expected to have lower accuracy. The Temporospatial (PCA/ICA) analysis was re-run for the 11 subjects with enough errors to include an average for error trials. Figure 5 illustrates that the $\mathrm{C} 1$ component amplitudes for the correct trials show a similar pattern as for the full sample. As predicted, the $\mathrm{C} 1$ amplitudes are markedly attenuated for error trials, consistent with the interpretation that the pre-stimulus negativity of $\mathrm{C} 1$ is a functional preparation for effective visual perception.

Source estimation for the grand-average of $\mathrm{C} 1$ showed prominent activity in the bilateral inferior temporal lobe (including the fusiform area), BA 18, and BA 7 (precuneus), and posterior cingulate cortex (PCC, see Figure 4C). The inferior temporal lobe activity was bilateral but stronger for the right hemisphere.

\section{Component 7 (C7)}

Figure 6 shows the waveforms and spatial topography of C7. This component also had two post-stimulus time intervals, one early $(88-156 \mathrm{~ms})$ and one late $(700+\mathrm{ms})$, that were sensitive to WM load. We analyzed this component using a repeated measures ANOVA with Task, Load, and Time (Interval 1, Interval 2) as within-subjects factors, referenced to the average of the -200 to $0 \mathrm{~ms}$ prestimulus interval. The adjusted significance level for multiple comparison is $p<0.005$. The main effects of Load, $F(1,16)=18.2, p<0.002$, and Time, $F(1,16)=37.7$, $p<0.001$, were significant, as was the Load $\times$ Time interaction, $F(1,16)=18.2, p<0.002$ (see Table 1). Paired $t$-tests showed 


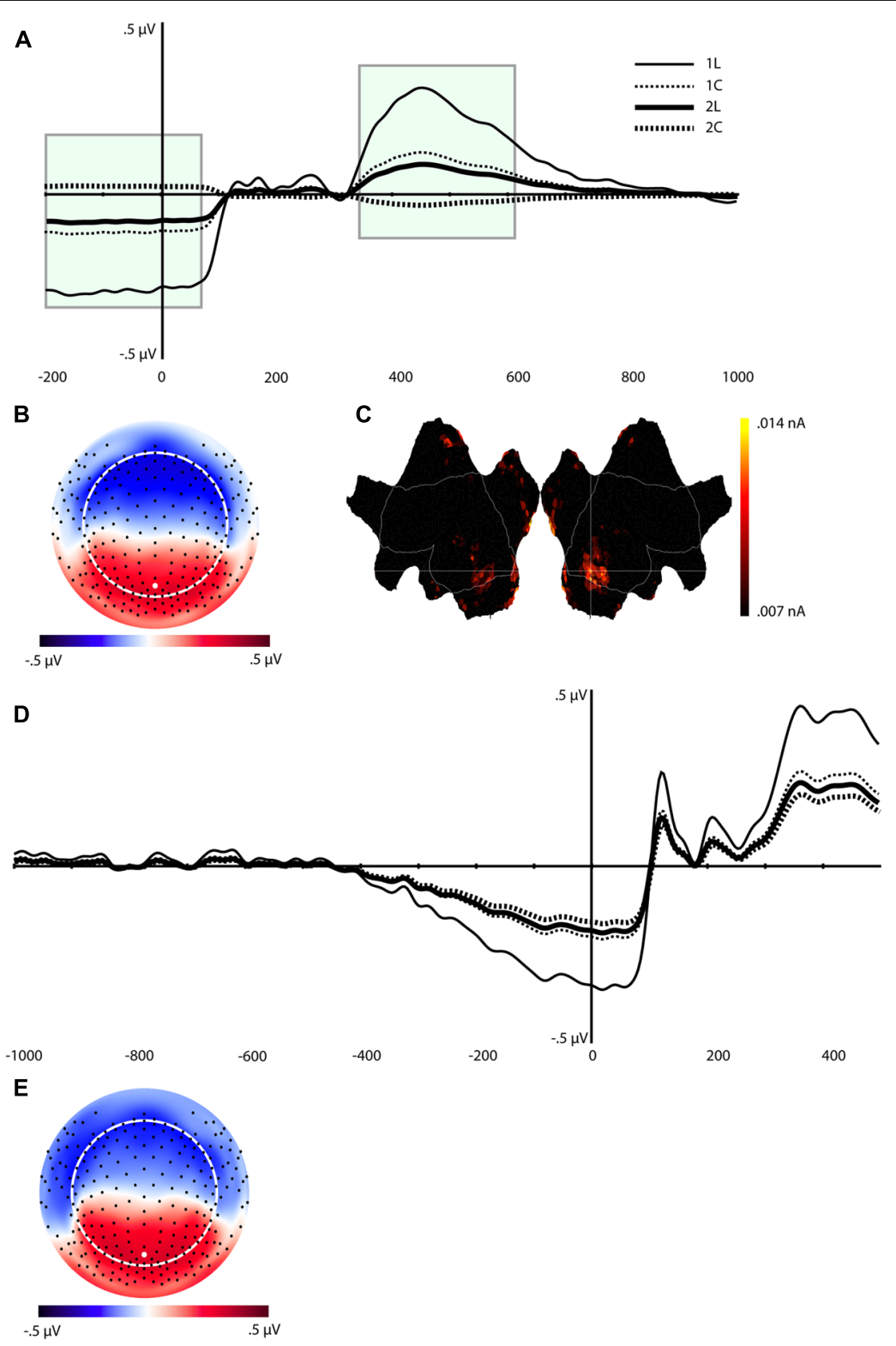

FIGURE 4 | Component (C1). (A) Component waveform. Green boxes represent the time windows used for statistical analysis. (B) Topographic map showing voltage distribution of $\mathrm{C} 1$. Orientation is top looking down with nose at the top. Positive voltage values are red and negative values are blue, with the zero crossing in white. Dots on the map are channel locations and white dot represents channel used for the waveform in (A).

(C) Source results projected onto a schematic flat map (unfolded cortex) to show activity at all cortical sites. The area within the white border represents the lateral surface (outside of this is the medial wall); the left side is the left hemisphere. Activity was thresholded to show the top 10\% of source activity. (D) This component waveform was re-computed with temporospatial analysis using a long prestimulus interval [see Component 1 (C1)]. (E) Topographic map showing voltage distribution, with the same topography as when computed with a shorter baseline. This component also showed a similar pattern of cortical source activity as the $\mathrm{C} 1$ in Figure 4C that at both time intervals the waveforms differed significantly, $t(16)=-2.3, p<0.04$ (early), $t(16)=2.3, p<0.04$ (late).

Source estimation results showed that, in addition to the extrastriate areas that we observed for $\mathrm{C} 1, \mathrm{C} 7$ included activation in the left middle frontal gyrus (MFG, BA 10), left inferior frontal gyrus (IFG, BA 46), medial orbitofrontal cortex (OFC, peak at BA 11 ), and temporal poles (stronger for the right hemisphere, see Figure 6).

\section{Component 9 (C9)}

This component was most prominent at approximately $350 \mathrm{~ms}$ post-stimulus (see Figure 7, C9) and resembles the P2 component and is affected by the load manipulation as previously reported (see Discussion). C9 was quantified as the mean activity $280-430 \mathrm{~ms}$ post-stimulus and referenced to the mean of the $200 \mathrm{~ms}$ prestimulus interval. Analysis included Task and Load factors. The main effects were significant - Task, $F(1,16)=18.2, p<0.002$, 
Table 1 | Summary table of components and experimental effects.

\begin{tabular}{|c|c|c|c|c|}
\hline Component & Source location & $\begin{array}{l}\text { Talairach coordinates of } \\
\text { maximum current density }\end{array}$ & Effect & Significance \\
\hline \multirow{2}{*}{$\mathrm{C} 1$} & & & Task & $p<0.04$ \\
\hline & & & Time & Ns \\
\hline \multirow[t]{4}{*}{$\mathrm{C} 7$} & Bilateral inf. temp. lobe, bilateral temp. poles, & $-3,45,-20$ & Load & $p<0.002$ \\
\hline & BA 18, BA 10, BA 11, BA 46 & & Task & Ns \\
\hline & & & Time & $p<0.001$ \\
\hline & & & Time $\times$ load & $p<0.002$ \\
\hline \multirow[t]{3}{*}{$\mathrm{C9}$} & Bilateral inf. temp. lobe, bilateral temp. poles, & $60,-53,-13$ & Load & $p<0.002$ \\
\hline & BA 18, middle and superior temporal gyri, BA & & Task & $p<0.002$ \\
\hline & 10, BA 11, BA 40 & & Load $\times$ task & Ns \\
\hline
\end{tabular}

PCC, posterior cingulate cortex.

and Load, $F(1,16)=15.7, p<0.002$ - confirming that the amplitude was largest in the 2-back and in the location conditions (see Table 1).

Source estimates were similar to $\mathrm{C} 1$. In fact, the location with maximal current density is the same as C1 (see Table 1). However, C9 also included activity in the middle and superior temporal gyri and in the inferior parietal lobe (BA 40, stronger in the left hemisphere, Figure 7C, C9) not present in C1. C9 also had sources that were similar to $\mathrm{C} 7$, notably activity at the temporal poles and medial prefrontal cortex.

\section{Network dynamics}

Together, the three components described above capture the essential aspects, both in terms of neural regions, as well as in time course of activity, of an overarching network that supports WM in the $n$-back test. To illustrate the sequence of component activations, Figure 8 plots the time courses, with insets of cortical activation patterns, for all of the components that varied significantly as a function of memory load. The underlying dynamics of this network seem to follow this time course:

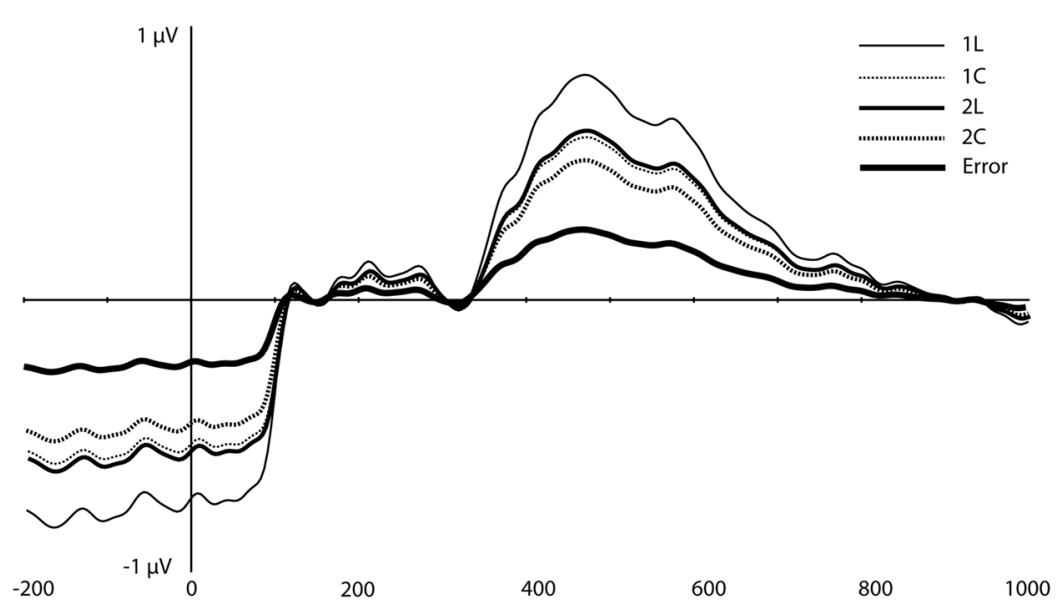

FIGURE 5 | Component 1 (C1) waveforms with error trials. 


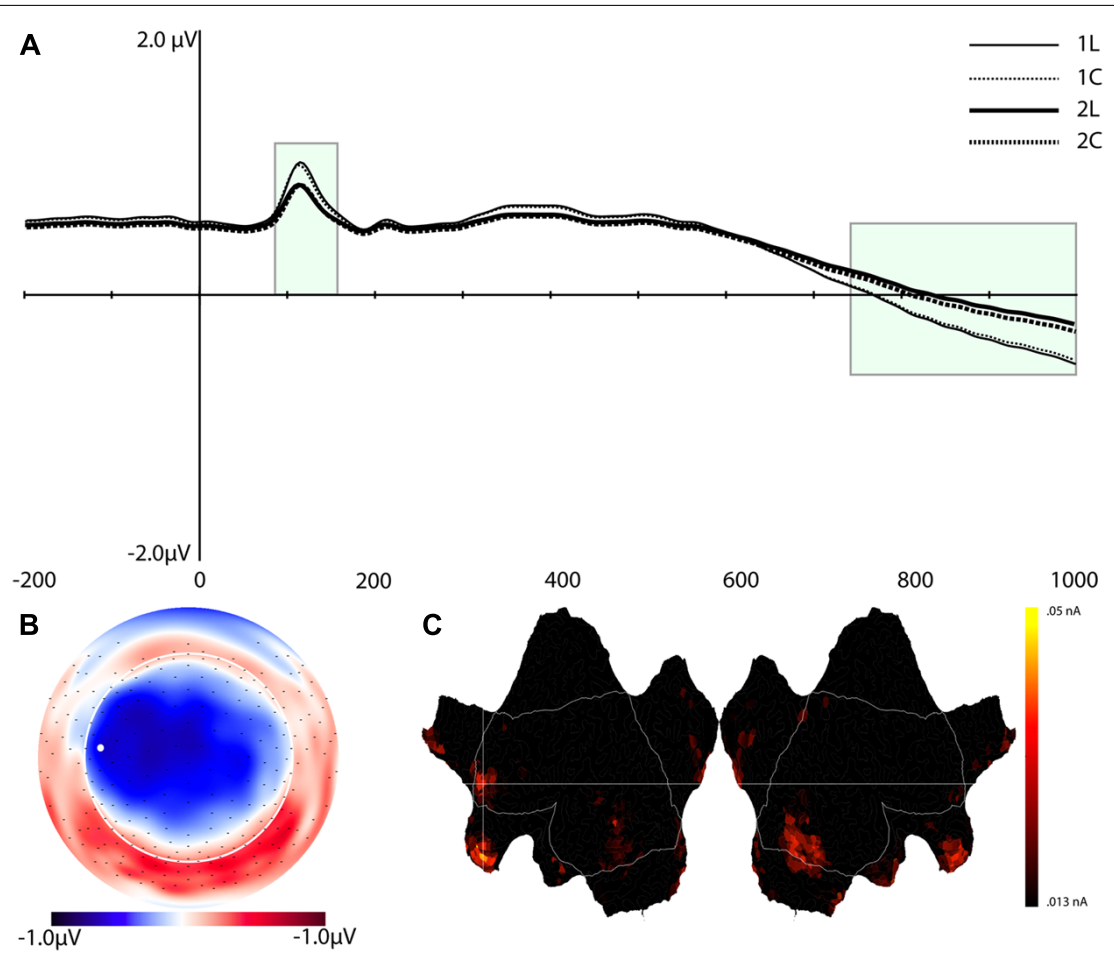

FIGURE 6 | Component 7 (C7). (A-C) are as in Figure 4.

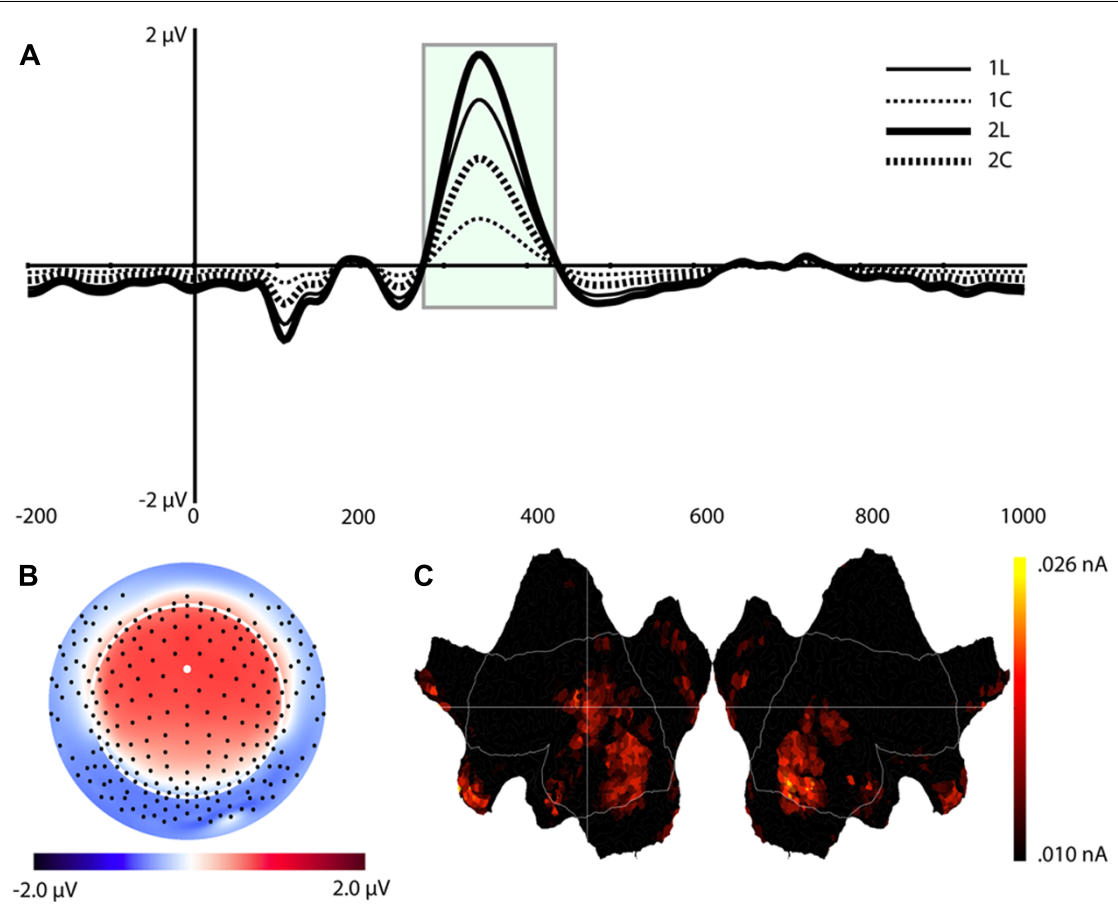

FIGURE 7 | Component $\mathbf{9}$ (C9). For each component, (A-C) are as in Figure 4 


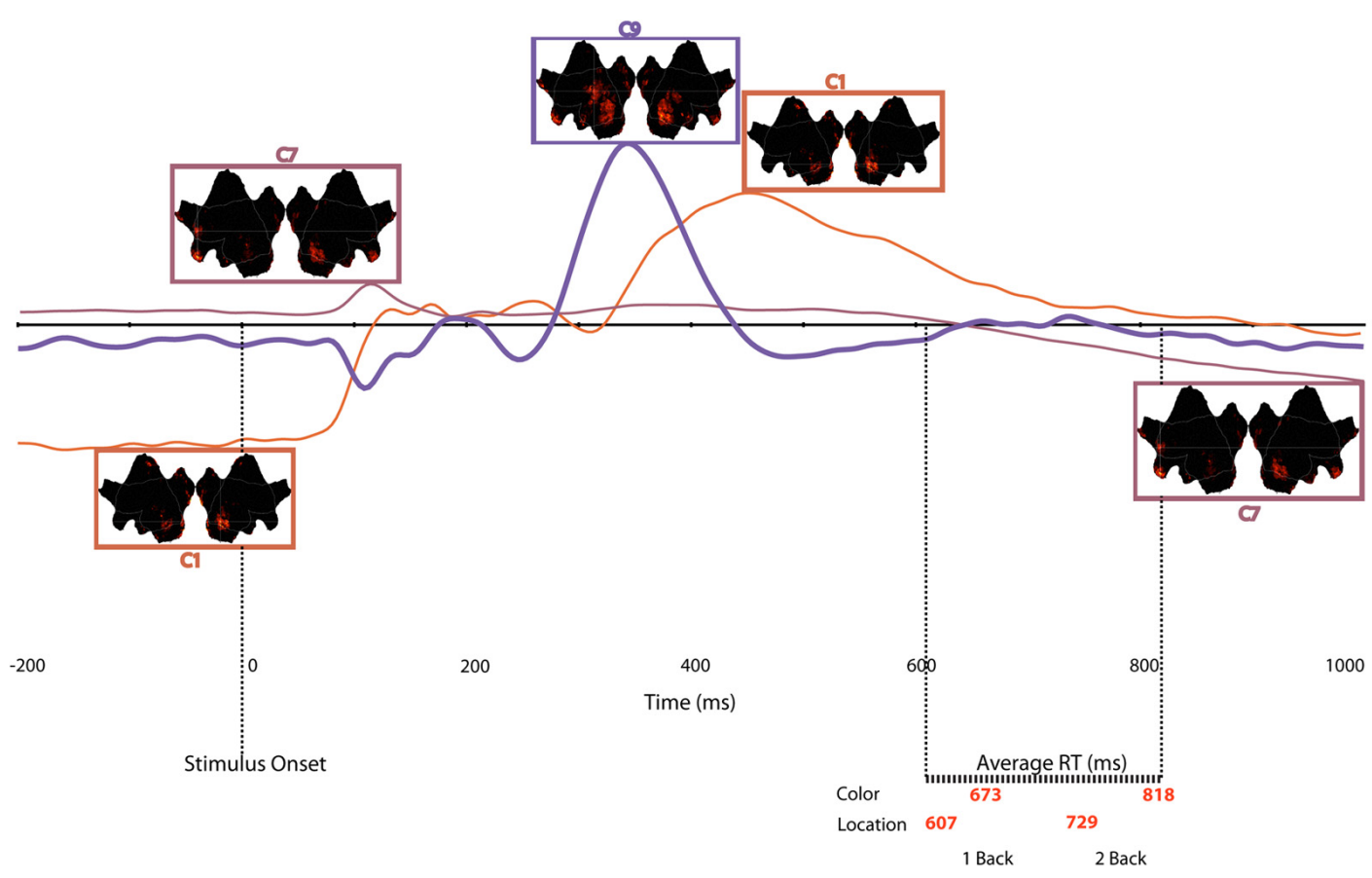

FIGURE 8 | Time course of each temporospatial component in relation to stimulus onset and mean reaction times for each experimental condition. Note that border of source maps are color coded to match waveforms.

(a) Engage active control (C1), $200 \mathrm{~ms}$ before stimulus onset to $80 \mathrm{~ms}$ post-onset

(b) Prime attentional resources (C7), $88 \mathrm{~ms}$ post-stimulus onset to $156 \mathrm{~ms}$ post-onset

(c) Focus attentional resources (C9), $280 \mathrm{~ms}$ post-stimulus onset to $430 \mathrm{~ms}$ post-onset

(d) Release control (C1) 340-630 ms post-stimulus onset

(e) Re-prime for the next trial (C7), $700 \mathrm{~ms}$ post-onset

\section{DISCUSSION}

In this $n$-back WM task, the subjects attended to a relevant feature of the visual stimulus (color or location), held it in memory, and evaluated whether that feature matches the same feature category on either the next trial (1-back) or the trial after the next trial (2-back). RT analysis indicated that, whereas the color memory task was more difficult overall, the effect of the WM load manipulation was roughly equivalent for the color and location tasks. While the accuracy data showed a significant interaction between task type and task load, with a larger effect of load in the color task, this interaction was likely due to a combination of the main effect of task with a ceiling effect in the 1-back conditions - participants were at or near ceiling in terms of accuracy for 1-back for both the color (98\% correct) and the location ( $98.4 \%$ correct) conditions. Nonetheless, RT was faster for the location 1-back condition (mean RT $=607 \mathrm{~ms}$ ) than the color 1-back condition $($ mean RT $=673 \mathrm{~ms} ; F(1,16)=8.46, p<0.01)$.

Having validated the WM load manipulation in the behavioral data, we then examined the brain's electrical activity that varied with WM load. To separate the brain activity superimposed at the head surface from multiple cortical sources, we conducted a temporospatial analysis, in which a temporal PCA was followed by a spatial ICA. The temporal PCA identified unique time courses (temporal components) that suggested patterns of covariance in time computed over the spatial features of brain activity (variability over channels), the task demands (variability over conditions), and individual differences (variability over subjects). The subsequent spatial ICA decomposition showed only three temporospatial component that varied with memory load. Our reasoning was that the temporospatial decomposition - when examined in relation to WM load - may suggest how to statistically decompose the complex time course of cortical activity into functionally meaningful patterns (i.e., networks). In some cases, these patterns may indicate activity from a single, modular brain source, while in other cases, these patterns may indicate networks of cortical function that must be coordinated to achieve effective WM. The LORETA source estimation maps provide a data-driven approach that makes few assumptions regarding modular vs. distributed network accounts of temporospatial ERP components. We first discuss the possible meaning of each temporospatial component, and then consider them together as an ensemble of interacting networks.

\section{TIME COURSES AND SPATIAL PATTERNS OF CORTICAL ACTIVITY IN WORKING MEMORY \\ Component C1}

Component $\mathrm{C} 1$ peaked at $456 \mathrm{~ms}$ post-stimulus, similar to the LPC. The memory load effect for C1 replicates load effects for the LPC reported by other researchers (Gevins et al., 1996; McEvoy 
etal., 1998). PCA studies show that the LPC can be decomposed into several components with unique topographies and time courses (Dien et al., 2004; Foti et al., 2007). One component identified by Foti et al. (2007) has an occipital distribution and a time course that seem to parallel C1. The apparent network pattern of $\mathrm{C} 1$, including both visual association areas and a more general (PCC) limbic cortex control function, is consistent with a major component of the LPC.

However, the $\mathrm{C} 1$ component in the present study also showed a pre-stimulus negativity that exactly mirrored the post-stimulus positivity of the apparent LPC starting at 350-400 ms. This pre-stimulus negativity is not typically attributed to the LPC in the literature. This may be due to the fact that for conventional ERP analysis, the typical practice of pre-stimulus baseline correction may mask the relationship between any pre-stimulus negativity and the LPC response. In the early literature on the P300 (a component closely related to the LPC), there was a controversy over whether the P300 could be interpreted as a "release" of the contingent negative variation (CNV). The CNV is a pre-stimulus negativity that appears to reflect preparation for processing, and perhaps for responding to, the stimulus (Williamson et al., 1977). Although Donchin and Heffley (1979) used factor analytic evidence to argue persuasively that the P300 and the CNV are distinct components, the present findings suggest that pre-stimulus negativity may figure importantly in P300like activity under certain task demands, such as placing explicit demands on WM. The pre-stimulus load effects on C1 are consistent with prior studies with the $n$-back task. McEvoy et al. (1998) observed WM load effects on CNV, and the time course of these effects was similar to that of the $\mathrm{C} 1$ component in the present study.

The CNV, initially described as the E wave or expectancy wave (Williamson et al., 1977), was later decomposed into two components: an early component reflecting an orienting response, and a late motor preparation response. More recent findings show that the late component of the CNV can be decomposed further into those components that reflect motor preparation and those that reflect anticipation of the arrival of sensory stimuli (Brunia and van Boxtel, 2001). The expectation for the sensory perception is most relevant to the present $\mathrm{C} 1$, which showed pre-stimulus negativity over visual association areas. This perceptual readiness component has been described as the SPN. Brunia and van Boxtel, 2001 presented evidence that the SPN reflects anticipatory attention that elicits sensory-specific cortical activation and reasoned on neuroanatomical grounds that the SPN, although generated in cortex, reflects control influences from the pulvinar nucleus of the thalamus. In the current study, C1 was generated in visual cortices (particularly on the right side) and in PCC, consistent with prior research showing that sources of the late CNV interval seem to engage the PCC, lateral and medial occipital cortex, and frontal regions (Gómez et al., 2003). Somewhat in contrast with prior work, however, C1 was separated from frontal regions, and the PCC was the apparent limbic control point for maintaining the priming in visual association cortices. Locally, the functional priming of visual cortex may be achieved through modification of the cortical depolarization threshold, manifesting as a surface negative potential (Rockstroh et al., 1993). Such an effect may be achieved through subcortical regulatory input from the pulvinar nucleus of the thalamus (Brunia and van Boxtel, 2001).

It may seem counterintuitive that the apparent priming of visual cortex was greater for the low load than the high load condition. A similar effect was also observed by McEvoy et al. (1998). This effect might indicate that for the low load condition the perceptual representation (and priming negativity or SPN) is maintained into the next trial, whereas for the high load condition the target perceptual representation must be re-accessed and is not maintained in the pre-stimulus interval.

Results from fMRI research are consistent with this interpretation that there is local priming of visual cortex when the representation of a visual stimulus is held in a perceptual store. Kastner et al. (1999) showed that when attention was allocated to a location that would eventually contain a visual stimulus, the visual cortices showed increased activity during the expectant period, even when no stimulus was present. Esterman and Yantis (2010) observed similar effects within object-selective regions of temporal cortex when participants were cued to anticipate a particular category of visual stimulus. Consistent with the interpretation that a greater priming negativity (SPN) reflects greater anticipatory attention, the amplitude of $\mathrm{C} 1$ in the present study varied with $\mathrm{RT}$, with larger $\mathrm{C} 1 \mathrm{~s}$ and faster responses not only for the low load vs. high load, but also for the location vs. color conditions.

A similar reasoning can be applied to the post-stimulus interval, where $\mathrm{C} 1$ peaked at $456 \mathrm{~ms}$ and mirrored the distribution of amplitudes over experimental conditions seen for the pre-stimulus interval. The response of $\mathrm{C} 1$ in the LPC interval was inversely related to the level of pre-stimulus priming. Although the $\mathrm{C} 1$ response in the LPC interval may reflect an active control mechanism, this activity might also reflect a kind of releasing mechanism, wherein the activity of the visual cortex is released from the control of attention circuits (such as the pulvinar, or perhaps the prefrontal cortex or temporoparietal junction - TPJ).

It is important to note that the $\mathrm{C} 1$ response is not an early visual response such as indexed by the P1 or N1. Those components were not affected by load, either in the present study or in previous studies (McCollough et al., 2007). Rather, the poststimulus $\mathrm{C} 1$ is a late response in visual association cortices and in memory-related limbic structures (PCC). Furthermore, the apparent network indexed by the $\mathrm{C} 1$ is not the classical P3b network the $\mathrm{C} 1$ topography differs substantially from the traditional P3b topography (see Foti et al. (2007), even though it does share the PCC as a pivotal source with the P3b (see Luu et al., 2007).

Considering both time windows of the $\mathrm{C} 1$, this component seems to reflect a functional priming and then functional activation of the visual perceptual network. If so, then we would expect an attenuated $\mathrm{C} 1$ (poor expectant priming) on trials with errors. In fact, a weak pre-stimulus $\mathrm{C} 1$ should predict a high likelihood of an error on that trial. Figure 5 shows the data for subjects with sufficient errors to create ERP averages for errors as well as for correct responses. The pattern of results for correct trials is similar to the analysis of the full sample. Furthermore, as predicted there is an attenuated $\mathrm{C} 1$ component for error trials. 


\section{Component $\mathrm{C7}$}

This component (Figure 6) reflects a significant enhancement for the low load vs. high load conditions early in the post-stimulus epoch, then apparently releasing slowly. To our knowledge this component has not been reported in the literature. Source analysis included right-lateralized activity in visual association cortex, similar to the area primed by $\mathrm{C} 1$, as well as right-lateralized temporal pole sources, perhaps consistent with the spatial demands of the task. In fMRI studies, temporal pole activation has been related to holistic perception of objects (Zhou et al., 2010). Furthermore, multiple structures of the anterior temporal lobe (superior, middle, and inferior temporal gyrus, perirhinal and entorhinal cortices, and uncus) have been shown to form a functional network involved in declarative memory processes (Gour et al., 2011).

Whereas these sources seem clearly relevant to the rightlateralized processing of the figural stimuli of this task, C7 was strongly left-lateralized for the sources in the frontopolar (MFG, IFG, and OFC) regions. The MFG and IFG have been identified in previous research as integral to a stimulus-driven ventral attention network (Corbetta et al., 2008). According to Corbetta et al. (2008), the ventral attention network is engaged by the appearance of important stimuli, independent of the salience of the stimuli. Specialized for object attention and memory, the ventral attention network seems to be particularly well developed in the left hemisphere, which supports analytic perception and object memory (Tucker et al., 2007; Tucker and Luu, 2012). Engagement of the OFC may be expected for the ventral attention network as well, particularly as it contributes to WM performance. Recent evidence shows that lesions to the OFC impair coordination of multiple discrete cognitive processes in the $n$-back task, such as simultaneous maintenance, manipulation, and monitoring of information (Barbey et al., 2011).

Based on fMRI studies, the control of the (right-lateralized) visual association areas (such as seen in C7) might be expected to engage the frontal networks of the (left-lateralized) ventral attention system (also seen in C7). However, the time course of this C7 component is remarkably early, with an abrupt peak at $\sim 100 \mathrm{~ms}$ as the earliest feature of the visual ERP in this study. On the surface, our current results, suggesting that frontal networks are engaged early, runs counter to the ERP literature, which shows that such early neuronal responses are typically observed over sensory cortex. How could frontal networks be engaged so early?

One possibility is that frontal attention control is engaged through non-specific thalamic projections in parallel with the sensory-specific thalamic projections to primary visual cortex. The frontal pole (reflected in the activity of C7) is particularly important to the thalamic regulation of attention, suggesting the possibility of two-way frontothalamic interactions in the early stages of attentional control. The frontal polar cortex in primates is connected to the anterior thalamic reticular nucleus (TRN, Zikopoulos and Barbas, 2006), which regulates thalamocortical activity (including sensory traffic). This anatomical organization puts the TRN, and its frontopolar cortical network, in a central position to regulate selective attention processes (Yingling and Skinner, 1997), as well as the general state of alertness (Tucker et al., 2007). A now classic hypothesis (Crick, 1994) proposes that the TRN's inhibitory control over thalamocortical projections provides the neural mechanism for the spotlight of attention. Furthermore, recent evidence shows that seizure discharges in the frontal pole are observed in absence spells with a momentary lapse in conscious attention (Tucker et al., 2007). In support of this proposal, early post-stimulus attentional selection-related processing has been observed in monkey PFC (Hasegawa et al., 2000; Everling et al., 2002).

Whatever the neural mechanism, the time course of $\mathrm{C} 7$ suggests that the component is re-engaged late in the epoch (Figure 6). That this activity returns after a response is made may suggest that there is attention modulation of the same areas in anticipation of the next trial, given the evaluation of present stimulus in the context of the 1-back or 2-back memory requirements.

\section{Component $C 9$}

Previous ERP research has produced somewhat contradictory results on WM load effects within this time window. Gevins et al. (1996) showed that a vertex-positive component occurring at approximately $200 \mathrm{~ms}$ post-stimulus was larger in high-load than in low load conditions for matching stimuli. In contrast, McEvoy et al. (1998) showed no effect of load for matching stimuli in the vertex P250, but P250 was larger for a spatial task compared to a verbal task.

At the scalp, C9 in the present study showed a peak positivity near the vertex (see Figure 7) at roughly the same time as the P200/P250 previously reported (Gevins et al., 1996; McEvoy et al., 1998). Like the P200/P250, the present C9 showed effects both of load (larger for high load, as reported by Gevins et al., 1996) and of task (larger for location compared to color, as reported by McEvoy et al., 1998). The conflicting results of load and task effects found by Gevins et al. (1996) and McEvoy et al. (1998) might be due to differences in experimental procedures, the use of different stimuli, quantification errors of the P200/P250 because of temporal overlap of different underlying components, or the use of surface Laplacian measures that are only sensitive to superficial cortical sources. In our analysis, the PCA/ICA procedure statistically separated C9 from other overlapping components, making its quantification more discrete.

We consider C9 to reflect the activity, engaged strongly at this time interval, of the ventral network identified by Corbetta et al. (2008). Whether functionally separable or not, these components reflect the importance of the posterior aspect of the superior temporal gyrus, occipital cortex, and the inferior parietal lobe in the control of attention in this WM task.

As noted by Corbetta et al. (2008), previous research has shown that activity in the TPJ is deactivated when participants have to keep more information in mind. This effect was interpreted as reflecting the suppression (by a dorsal, goal/task oriented network) of the reorienting network to enable memory traces to be maintained during a retention interval. Consistent with this reasoning, they noted that during high-workload conditions, novel unattended stimuli are less likely to be detected. Chambers et al. (2004) also showed, using transcranial magnetic stimulation, that when the right TPJ is stimulated between $90-120 \mathrm{~ms}$ and $210-240 \mathrm{~ms}$ after stimulus onset, reorienting to a new stimulus is impaired. 
They argued that the later effect reflects a different stage of processing within the slower visual pathway. The slower time course identified by Chambers et al. would place that effect at about the onset of the present C9 component.

\section{TIME COURSE OF MULTIPLE NETWORKS IN WORKING MEMORY}

Considering the multiple load-dependent components together, the temporospatial analysis revealed networks (statistically defined by PCA and ICA) that span a pre-stimulus preparation to postresponse consolidation interval (Figure 8). Across time, three networks emerged, with each network sharing some common cortical nodes and, critically, involving unique, non-shared nodes as well (see Table 1). The first network to appear in the data involves bilateral visual association areas and the PCC. The pre-stimulus negativity of $\mathrm{C} 1$ seems to reflect priming of visual cortex for cognitive processing of the trial through an anticipatory attention mechanism. Consistent with this notion, fMRI studies have shown that the PCC is part of the DMN and that greater PCC deactivation is associated with faster reaction times (Chadick and Gazzaley, 2011). Similarly, Anticevic et al. (2010) showed that, compared to error responses, correct responses were associated with greater deactivation of the DMN. In the present study, although we have considered the PCC as providing executive control over visual priming (Mesulam, 1981), it might be important to consider the alternative that the PCC element of the pre-stimulus C1 may reflect suppression of the PCC and thus DMN in order to support the perceptual priming or memory maintenance required for task performance.

Shortly after stimulus onset, the C1 network appears to be released, and then at about $100 \mathrm{~ms}$ there is abrupt activity in all other time course components that reflect memory load effects. One of these components (C7) shows a brief peak at about the same time as $\mathrm{C} 1$ activity diminishes and then goes quiescent until a slow opposing ramp late in the epoch. The cortical network engaged by $\mathrm{C} 7$ seems to reflect recruitment of attentional control (left frontopolar), holistic memory representation (right anterior temporal), and priming of visual association areas. Even though it is engaged just briefly, we speculate that this C7 network sets the stage, through top-down control, for further processing.

It is important to point out familiar ERP components, $\mathrm{P} 1$ and N1, which are missing from Figure $\mathbf{8}$ because they do not vary with WM load. Some later scalp topographic features of the conventional N1 may be discerned in the map of the C9 component (Figure 7), but these are posterior negative inversions of the familiar frontal P2 reflected by $\mathrm{C}$, and are not reflective of the primary N1 component. Thus, although some of the sources of activity noted across the various components reported here bear similarity to cortical nodes activated during selective attention tasks, the temporal effects of WM load on neural processes appear to occur later than the earliest effects reported for spatial (Martinez et al., 1999) and color-based (Zhang and Luck, 2008) attentional selection tasks.

In the conventional P2 and early P3 interval of the ERP (280-430 ms), a set of networks show load effects. C9 reflects the familiar anterior centrofrontal P2 of the ERP, and one interpretation is that it re-engages networks previously primed or activated by the $\mathrm{C} 1$ and $\mathrm{C} 7$ components. Certain structures disappeared (such as the lateral MFG and IFG seen in C7) and new ones were added (such as the TPJ in C9). This P2/C9 network may reflect attentional modulation, through regulation of orienting responses to the presented stimulus, and it involves not only sensory areas but also the OFC, which is active when attention must reorient following invalid cues (Nobre et al., 1999) and the temporal poles. After the 280-430 ms interval, the C1 network reappears to contribute to the later LPC (Dien et al., 2004; Foti et al., 2007). Finally, C7 ramps in an opposite direction to its initial deflection late in the epoch after responses have been made. Perhaps this suggests that the same processes involved in preparing processing for the stimulus on a current trial are re-engaged to prepare processing on the next trial.

With the exception of the $\mathrm{C} 1$ component, the cortical regions engaged by $\mathrm{C} 7$ and $\mathrm{C} 9$ reflect temporospatial networks of the ventral attention network identified by Corbetta et al. (2008). The temporal resolution of dEEG allowed us to delineate how the component networks of the ventral attention system, such as the MFG, IFG, and TPJ, emerge at different points in time. Consistent with the reactive (stimulus-initiated) nature of the ventral attention system, C7 and C9 were reactive in the post-stimulus interval.

\section{LIMITATIONS OF THE PRESENT STUDY}

This study was designed to manipulate WM load parametrically, through the $n$-back task. However, the conventional cognitive psychology interpretation of this task, as a memory process that must be held in an active store for a short time or a longer time, may be incorrect. Particularly when observing the dEEG evidence that there was priming of visual association areas, and that this was most strong for the 1-back condition, it seems apparent that the 1-back condition is a different cognitive task, which involves holding a percept in an active visual store, than the 2-back condition, which does not involve maintenance of a perceptual store (which is impossible because of the intervening stimulus) but rather requires a different (perhaps more abstract or symbolic) access of the 2-back stimulus. This finding could also be accommodated by an account grounded in dual-process theories of memory, in which declarative and non-declarative representational systems contribute in varying proportions to $n$-back performance and in which declarative processes play a greater role under 1-back conditions than under 2-back conditions (Caggiano et al., 2006; Vilberg and Rugg, 2008). Further research is required to determine if the difference between the 1-back and 2-back conditions is indeed qualitative, as seems in the present study, rather than a quantitative difference in memory load.

Although the $n$-back task is commonly used to study WM processes, the component processes (such as storage, maintenance, and executive control) are not readily separable. Our interpretation of the functional significance of each component not only relied on the task effects and time course, but also previous findings. Therefore, our interpretations must be tested in future research, using paradigms that can separate different WM processes, such as storage, rehearsal, and executive processes (e.g., Kiss et al., 2007).

The use of the WM load manipulation to identify functionally relevant components in the temporospatial analysis caused us to select certain components from the larger set of temporospatial components that are important to understand the cognitive 
processes common to the two memory load conditions. Future research should not only examine which of the present components replicate clearly, but also examine these in light of the complete set of time courses and cerebral networks required for all elements of task performance.

\section{CONCLUSION}

The present study suggests that a temporospatial analysis can separate several unique time courses of neural activity with different underlying cortical networks that contribute to WM performance. In recent cognitive models, WM is considered to be served by top-down, temporary activation of items in long-term memory (Ruchkin et al., 2003) via directed attention (e.g., Cowan, 1997; Postle, 2006). The present results suggest that temporospatial analysis of the ERP can isolate the contribution of key nodes in the frontal lobe, TPJ, and PCC that appear to mediate directed attention. Nonetheless, the cortical activity most clearly related to this visual WM task was not an abstract representation from longterm memory, but the priming of visual cortex that seemed to reflect preparation for a direct match between the representational expectancy and the visual percept.

\section{AUTHOR CONTRIBUTIONS}

Phan Luu, Daniel Caggiano, Alexandra Geyer, and Jenn Lewis contributed to the design of the study. Jenn Lewis was responsible for data acquisition. Phan Luu, Daniel Caggiano, Alexandra Geyer, Jenn Lewis, and Don Tucker contributed to data analysis. All authors contributed to the interpretation and preparation of the manuscript. All authors approve the version that is currently under consideration and acknowledge that they are accountable for all aspects the work.

\section{ACKNOWLEDGMENT}

This research is funded by a grant from the Office of Naval Research.

\section{REFERENCES}

Anticevic, A., Repovs, G., Shulman, G. L., and Barch, D. M. (2010). When less is more: TPJ and default network deactivation during encoding predict working memory performance. Neuroimage 49, 2638-2648. doi: 10.1016/j.neuroimage.2009.11.008

Barbey, A. K., Koenigs, M., and Grafman, J. (2011). Orbitofrontal contribution to human working memory. Cereb. Cortex 21, 789-795. doi: 10.1093/cercor/ bhq153

Brunia, C. H. M., and van Boxtel, G. J. M. (2001). Wait and see. Int. J. Psychophysiol. 43, 59-75. doi: 10.1016/S0167-8760(01)00179-9

Caggiano, D. M., and Parasuraman, R. (2004). The role of memory representation in the vigilance decrement. Psychon. Bull. Rev. 11, 932-937. doi: 10.3758/BF03196724

Caggiano, D. M., Jiang, Y., and Parasuraman, R. (2006). Aging and repetition priming for targets and distracters in a working memory task. Aging Neuropsychol. Cogn. 13, 552-573. doi: 10.1080/138255890969555

Chadick, J. Z., and Gazzaley, A. (2011). Differential coupling of visual cortex with default or frontal parietal network based on goals. Nat. Neurosci. 14, 830-832. doi: $10.1038 / \mathrm{nn} .2823$

Chambers, C. D., Payne, J. M., Stokes, M G., and Mattingley, J. B. (2004). Fast and slow parietal pathways. Nat. Neurosci. 7, 217-218. doi: 10.1038/nn1203

Corbetta, M., Patel, G., and Shulman, G. L. (2008). The reorienting system of the human brain: from environment to theory of mind. Neuron 58, 306-324. doi: 10.1016/j.neuron.2008.04.017

Cowan, N. (1997). Attention and Memory: An Integrated Framework. Cambridge: Oxford University Press.
Crick, F. (1994). The Astonishing Hypothesis: The Scientific Search for the Soul. New York: Charles Scribner' Sons.

Dien, J. (2010). The ERP PCA toolkit: an open source program for advance statistical analysis of event-related potential data. J. Neurosci. Methods 187, 138-145. doi: 10.1016/j.jneumeth.2009.12.009

Dien, J. (2012). Applying principal components analysis to event-related potentials: a tutorial. Dev. Neuropsychol. 37, 497-517. doi: 10.1080/87565641.2012. 697503

Dien, J., Spencer, K. M., and Donchin, E. (2004). Parsing the late positive complex: mental chronometry and the ERP components that inhabit the neighborhood of the P300. Psychophysiology 41, 665-678. doi: 10.1111/j.1469-8986.2004.00193.x

Donchin, E., and Heffley, E. F. (1979). The independence of the P300 and the CNV reviewed: a reply to Wastell. Biol. Psychol. 9, 177-188. doi: 10.1016/03010511(79)90038-3

Druzgal, T. J., and D'Esposito, M. (2001). Activity in fusiform face area modulated as a function of working memory load. Cogn. Brain Res. 10, 355-364. doi: 10.1016/S0926-6410(00)00056-2

Esterman, M., and Yantis, S. (2010). Perceptual expectation evokes category-selective cortical activity. Cereb. Cortex 20, 1245-1253. doi: 10.1093/cercor/bhp188

Everling, S., Tinsley, C. J., Gaffan, D., and Duncan, J. (2002). Filtering of neural signals by focused attention in the monkey prefrontal cortex. Nat. Neurosci. 5, 671-676. doi: 10.1038/nn874

Ferree, T., Eriksen, K. J., and Tucker, D. M. (2000). Region head tissue conductivity estimation for improved EEG analysis. IEEE Trans. Biomed. Eng. 47, 1584-1592. doi: $10.1109 / 10.887939$

Foti, D., Hajcak, G., and Dien, J. (2007). Differentiating neural responses to emotional pictures: evidence from temporal-spatial PCA. Psychophysiology 46 521-531. doi: 10.1111/j.1469-8986.2009.00796.x

Fox, M. D., Snyder, A. Z., Vincent, J. L., Corbetta, M., Van Essen, D. C., and Raichle, M. E. (2005). The human brain is intrinsically organized into dynamic, anticorrelated functional networks. Proc. Natl. Acad. Sci. U.S.A. 102, 9673-9678 doi: 10.1073/pnas.0504136102

Gevins, A., Smith, M. E., Le, J., Leong, H., Bennett, J., Martin, N., et al. (1996). High resolution evoked potential imaging of the cortical dynamics of human working memory. Electroencephalogr. Clin. Neurophysiol. 98, 327-348. doi: 10.1016/00134694(96)00288-X

Gómez, C. M., Marco, J., and Grau C. (2003). Preparatory visuomotor cortical network of the contingent negative variation estimated by current density. Neuroimage 20, 216-224. doi: 10.1016/S1053-8119(03) 00295-7

Gordon, E. M., Breeden, A. L., and Bean. S. E., and Vaidya, C. J. (2012). Working memory-related changes in functional connectivity persist beyond task disengagement. Hum. Brain Mapp. doi: 10.1002/hbm.22230 [Epub ahead of print].

Gour, N., Ranjeva, J.-P., Ceccaldi, M., Confort-Gouny, S., Barbeau, E., Soulier, E., et al. (2011). Basal functional connectivity within the anterior temporal network is associated with performance on declarative memory tasks. Neuroimage 58 687-697. doi: 10.1016/j.neuroimage.2011.05.090

Hampson, M., Driesen, N. R., Skudlarski, P., Gore, J. C., and Constable, R. T. (2006). Brain connectivity related to working memory performance. J. Neurosci. 26, 13338-13343. doi: 10.1523/JNEUROSCI.3408-06.2006

Hasegawa, R. P., Matsumoto, M., and Mikami, A. (2000). Search target selection in monkey prefrontal cortex. J. Neurophysiol. 84, 1692-1696.

Horn, J. L. (1965). A rationale and test for the number of factors in factor analysis. Psychometrika 23, 187-200.

Jaeggi, S. M., Buschkuehl, M., Perrig, W. J., and Meier, B. (2010). The concurrent validity of the n-back task as a working memory measure. Memory 18, 394-412. doi: 10.1080/09658211003702171

Kastner, S., Pinsk, M. A., De Weerd, P., Desimone, R., and Ungerleider, L. G. (1999). Increased activity in human visual cortex during directed attention in the absence of visual stimulation. Neuron 22, 751-761. doi: 10.1016/S0896-6273(00) 80734-5

Kirchner, W. K. (1958). Age differences in short-term retention of rapidly changing information. J. Exp. Psychol. 55, 352-358. doi: 10.1037/h0043688

Kiss, I., Pisio, C., Francois, A., and Schopflocher, D. (1998). Central executive function in working memory: event-related brain potential studies. Cogn. Brain Res. 6, 235-247. doi: 10.1016/S0926-6410(97)00035-9 
Kiss, I., Watter, S., Heisz, J. J., and Shedden, J. M. (2007). Control processes in verbal working memory: an event-related potential study. Brain Res. 1172, 67-81. doi: 10.1016/j.brainres.2007.06.083

Lancaster, J. L., Woldorff, M. G., Parsons, L. M., Liotti, M., Freitas, C. S., Rainey, L., et al. (2000). Automated Talairach Atlas labels for functional brain mapping. Hum. Brain Mapp. 10, 120-131. doi: 10.1002/1097-0193(200007)10:3<120::AID$\mathrm{HBM} 30>3.0 . \mathrm{CO} ; 2-8$

Luu, P., Tucker, D. M., and Stripling, R. (2007). Neural mechanisms for learning actions in context. Brain. Res. 1179, 89-105. doi: 10.1016/j.brainres.2007.03.092

Martinez, A., Anllo-Vento, L., Sereno, M. I., Frank, L. R., Buxton, R. B., Dubowitz, D. J., et al. (1999). Involvement of striate and extrastriate visual cortical areas in spatial attention. Nat. Neurosci. 2, 364-369. doi: 10.1038/7274

McCollough, A. W., Machizawa, M. G., and Vogel, E. K. (2007). Electrophysiological measures of maintaining representations in visual working memory. Cortex 43 , 77-94. doi: 10.1016/S0010-9452(08)70447-7

McEvoy, L. K., Smith, M. E., and Gevins, A. (1998). Dynamic cortical networks of verbal and spatial working memory: effects of memory load and task practice. Cereb. Cortex 8, 563-574. doi: 10.1093/cercor/8.7.563

Mesulam, M. A. (1981). Cortical network for directed attention and unilateral neglect. Ann. Neurol. 10, 309-325. doi: 10.1002/ana.410100402

Newton, A. T., Morgan, V. L., Rogers, B. P., and Gore, J. C. (2011). Modulation of steady state functional connectivity in the default mode and working memory networks by cognitive load. Hum. Brain Mapp. 32, 1649-1659. doi: 10.1002/hbm. 21138

Nobre, A. C., Coull, J. T., Frith, C. D., and Mesulam, M. M. (1999). Orbitofrontal cortex is activated during breaches of expectation in tasks of visual attention. Nat. Neurosci. 2, 11-12. doi: 10.1038/4513

Owen, A. M., McMillan, K. M., Laird, A. R., and Bullmore, E. (2005). N-back working memory paradigm: a meta-analysis of normative functional neuroimaging studies. Hum. Brain Mapp. 25, 46-59. doi: 10.1002/hbm.20131

Pascual-Marqui, R. (2002). Standardized low resolution brain electromagnetic tomography (sLORETA): technical details. Methods Find. Exp. Clin. Pharmacol. 24D, 5-12.

Pessoa, L., Gutierrez, E., Bandettini, P. A., and Ungerleider, L. G. (2002). Neural correlates of visual working memory: fMRI amplitude predicts task performance. Neuron 35, 975-987. doi: 10.1016/S0896-6273(02)00817-6

Postle, B. R. (2006). Working memory as an emergent property of the mind and brain. J. Neurosci. 139, 23-38.

Rockstroh, B., Muller, M., Wagner, M., Cohen, R., and Elbert, T. (1993). "Probing” the nature of the CNV. Electroencephalogr. Clin. Neurophysiol. 87, 235-241. doi: 10.1016/0013-4694(93)90023-O

Ruchkin, D. S., Grafman, J., Cameron, K., and Berndt, R. S. (2003). Working memory retention systems: a state of activated long-term memory. Behav. Brain Sci. 26, 709-727. doi: 10.1017/S0140525X03000165

Ryynanen, O. R., Hyttinen, J. A., and Malmivuo, J. A. (2006). Effect of measurement noise and electrode density on the spatial resolution of cortical potential distribution with different resistivity values for the skull. IEEE Trans. Biomed. Eng. 53, 1851-1858. doi: 10.1109/TBME.2006.873744

Sala-Llonch, R., Peña-Gómez, C., Arenaza-Urquijo, E. M., Vidal-Piñeiro, D., Bargalló, N., Junqué, C., et al. (2012). Brain connectivity during resting state and subsequent working memory task predicts behavioural performance. Cortex 48 , 1187-1196. doi: 10.1016/j.cortex.2011.07.006
Tucker, D. M., Brown, M., Luu, P., and Holmes, M. D. (2007). Discharges in ventromedial frontal cortex during absence spells. Epilepsy Behav. 11, 546-557. doi: 10.1016/j.yebeh.2007.04.023

Tucker, D. M., and Luu, P. (2012). Cognition and Neural Development. New York: Oxford University Press.

Vilberg, K. L., and Rugg, M. D. (2008). Memory retrieval and the parietal cortex: a review of evidence from a dual-process perspective. Neuropsychologia 46, 1787 1799. doi: 10.1016/j.neuropsychologia.2008.01.004

Weber, T. A., Kramer, A. F., and Miller, G. A. (1997). Selective processing of superimposed objects: an electrophysiological analysis of object-based attentional selection. Biol. Psychol. 45, 159-182. doi: 10.1016/S0301-0511(96) 05227-1

Williamson, P. D., Allison, T., Goff, W. R., and Mattson, R. H. (1977). Evoked potential abnormalities in epilepsy: the E-wave. Electroencephalogr. Clin. Neurophysiol. 42, 729-730.

Yi, Y., and Friedman, D. (2011). Event-related potential (ERP) measures reveal the timing of memory selection processes and proactive interference resolution in working memory. Brain Res. 1411, 41-56. doi: 10.1016/j.brainres.2011. 07.004

Yingling, C. D., and Skinner, J. E. (1997). "Gating of thalamic input to cerebral cortex by nucleus reticularis thalami," in Progress in Clinical Neurophysiology: Attention Voluntary Contraction and Event-Related Cerebral Potentials, ed. J. E. Desmedt (Berlin: Basel-Karger), 70-96.

Zhang, W., and Luck, S. J. (2008). Feature-based attention modulates feedforward visual processing. Nat. Neurosci. 12, 24-25. doi: 10.1038/nn.2223

Zhang, Y., van Drongelen, W., and He, B. (2006). Estimation of in-vivo brainto-skull conductivity ratio in humans. Appl. Phys. Lett. 89, 223903. doi $10.1063 / 1.2398883$

Zhou, K., Luo, H., Zhou, T., Zhou, Y., and Chen, L. (2010). Topological change disturbs object continuity in attentive tracking. Proc. Natl. Acad. Sci. U.S.A. 107 21920-21924. doi: 10.1073/pnas.1010919108

Zikopoulos, B., and Barbas, H. (2006). Prefrontal projections to the thalamic reticular nucleus form a unique circuit for attentional mechanisms. J. Neurosci. 26 7348-7361. doi: 10.1523/JNEUROSCI.5511-05.2006

Conflict of Interest Statement: The authors declare that the research was conducted in the absence of any commercial or financial relationships that could be construed as a potential conflict of interest.

Received: 05 November 2013; accepted: 06 January 2014; published online: 29 January 2014.

Citation: Luu P, Caggiano DM, Geyer A, Lewis J, Cohn J and Tucker DM (2014) Timecourse of cortical networks involved in working memory. Front. Hum. Neurosci. 8:4. doi: 10.3389/fnhum.2014.00004

This article was submitted to the journal Frontiers in Human Neuroscience.

Copyright (c) 2014 Luu, Caggiano, Geyer, Lewis, Cohn and Tucker. This is an open-access article distributed under the terms of the Creative Commons Attribution License (CC BY). The use, distribution or reproduction in other forums is permitted, provided the original author(s) or licensor are credited and that the origina publication in this journal is cited, in accordance with accepted academic practice. No use, distribution or reproduction is permitted which does not comply with these terms. 